$\xi=-1$

\title{
2D-FEA Based Design and Performance Analysis of Low Weight Segmented Rotor HE-FSM for Light Weight Aircrafts
}

\author{
Hassan Ali, Erwan Sulaiman, Zamri Omar, M. F. Omar, Faisal Amin \\ Department of Electrical Power Engineering, \\ Department of Aeronautical Engineering \\ Universiti Tun Hussien Onn Malaysia, 86400 Parit Raja \\ *Email: engg.hassansoomro@gmail.com
}

\begin{abstract}
All electric aircraft (AEA), is one of the main intentions of the aerospace industry for future. Where electrical machines are capable to provide high torque density and are dominant for the feasibility of direct drive electrical driving force for aircraft applications. Besides, low weight and high torque capabilities, the best candidate solution should also inherently fault tolerant for aircraft applications. For these reasons, a new sort of machine has been familiarized and published in last decade know as flux switching machine (FSM). FSMs contain all excitation sources on stator side with robust rotor structure. According to the type of excitation, FSMs are characterized into three types such as permanent magnet PM FSM, field excitation FE FSM and hybrid excitation HE FSM. PM FSM and FE FSM use PM and FE coil for their excitation sources respectively, whereas both PM and FE coil is used in HE-FSM for excitation. Subsequently, these machines have shown high torque to weight ratios and high efficiency during research in the last decade. Therefore in this paper, a new structure of 12S-8P HE-FSM with segmental rotor has been proposed and analyzed. The proposed segmented HE-FSM has the simple structure using only three PMs and three FECs. The proposed structure is analyzed using commercial 2D FEA package, JMAG-designer ver. 14.0. This paper presents the coil test analysis of segmented HE-FSM to confirm the working principle. Besides, cogging torque, flux strengthening, torque vs current densities and power vs current densities have been analyzed and presented.

Keywords: aircraft applications; flux switching; hybrid excitation; segmental rotor; torque analysis.
\end{abstract}

\section{Introduction}

Inflight passenger transportation has been increasing annually at the rate of $9 \%$ since the 1960s and has brought the world nearer to the global warming [1]. While currently air transportation remains overpriced and accounts $2 \%$ of the artificial carbon dioxide $\left(\mathrm{CO}_{2}\right)$ emissions. Hence, it is the challenge for the aircraft operators as well as the aerospace industry to offer unremitting enhancements in terms of protection, reliability, safety, and availability while dropping cost, noise, and emission of $\mathrm{CO}_{2}$ [2]. To avail these prospects, aerospace systems are undergoing a long-term shift from mechanical, hydraulic, and pneumatic power systems into globally optimized electrical systems.

Electrical motor drives are reliable and capable of transferring electrical power to drive actuators, fuel pumps, air compressors and other subsystems at various speed ranges in conjunction with advanced power electronics and control schemes [3]. While meeting reliability requirements electric drives are able to gain overall efficiency and reducing the weight and cost. Therefore, it is the ultimate aim of the aircraft industry to achieve the "all-electric aircraft" (AEA), which converts all power systems to electrical power. It is estimated that an AEA can decrease aircraft weight and fuel consumption by $10 \%$ and $9 \%$ respectively [4].

Therefore, mechanically driven actuators have been gradually switched into electronic servo valve control "electro-hydraulic actuation hydraulic actuators." In the A380, electro-hydrostatic actuators have been used which provide hydraulic actuation by means of using pump and reservoir, and the operation took place by the electrical power supply. This "more electric" development has reduced the drawbacks of reduction in mechanical linkages. Currently, hydraulic power supply networks are easy and simple in terms of maintenance and less in weight than mechanical systems. Such as, electric engine has been used in fuel pumps, in place of hydraulic engine, which has been recognized to provide profits in system efficiency, weight, and size, and flexible in speed control [5]

To introduce the concept of electric actuators for more electric aircraft (MEA), electric motor and the control electronics both are kept in to account. In actual fact, for high performances, variable speed drives, the core of MEAs, are still far away to meet the high-reliability limitations needed in harmful conditions due to the presence of complex control systems employing power electronics, and VLSI microcontrollers or ASICs [6].

Although, significant improvements can be obtained to increase the reliability and performance of motor drives for the MEA, is present multi-phase machines. Multi-phase machines are generally intended as machines with a number of phases more than three, in the case of a one-phase fault, this number expected to be the critical threshold. In literature, a number of proposals have been found, reporting multi-phase motors nurtured by multi-phase power electronic converters, where both the motor and drive are designed to satisfy severe fault-tolerant requirements [7], [8]. A fault-tolerant design methodology differs from a pure redundant one in that provisions are made for planned degraded modes of operation where it is acceptable. By providing advantages for potential failures, a fault tolerant system may achieve reliability 
without recourse to non-optimized redundancy or over-sizing [9]. The significant areas, which can be taken as consideration of safety-critical drives for aircraft are engine generators, flight surface actuators, flap actuators, engine fuel pumps, and landing gear nose wheel steering systems [10].

In recent, a new form of the machine named as 'flux switching machine' (FSM) has been established and published which consists of active flux sources on the stator. In addition, besides the benefit of the brushless machine, it contains a single piece of an iron rotor which is robust, winding less, and efficient for highspeed applications. In FSM, the flux is controlled and maintained by the field flux. Moreover, there a three groups of FSMs, which are (i) permanent magnet PM-FSM, (ii) field excitation FE-FSM and (iii) hybrid excitation HE-FSM. PMs and FECs are key active flux sources of PM-FSM and FEFSM respectively. While both $\mathrm{PM}$ and FECs are combined to produce flux in HE-FSM [11-12].

Currently, PM-FSMs are being gradually more popular and widely used in several applications because of the advancement of modern high performances of rare earth magnetic materials. The applications where PM-FSMs are being widely used are electric and hybrid electric vehicles, renewable energy systems including wind power generators, industrial drives, electric aircraft, and automation, to domestic appliances [13].

Nevertheless, PM machines have some hitches, such as high cost of rare earth magnet, relatively lower flux-weakening capability, un-controllable flux, demagnetization and limited working temperature [14]. Therefore, non-PM machines have become one of the most widespread research topics. In [15], field excitation flux switching FE-FSM machine has been proposed and analyzed with the double stator. However, it draws more copper losses as both the armature and field windings are separately housed on the outer and inner stators. It also sacrifices torque density and efficiency due to less flux strengthening capabilities.

Hence, to combine the merits of PM-FSM and FE-FSM machines the PM and FE are coexist, and the concept of hybrid excited HE machines have been proposed. HE machines are admirable applicants for variable speed applications, such as electric vehicle, aircraft and wind power generation [16], [17]. There are a numerous number of topologies of HE machines have been introduced and published, because of two excitation sources PM and FE can be arranged in various techniques and arrangements. Hence several numbers of new designs and structures have been proposed and investigated in the past two decades. According to the structure and construction between FE and PM fluxes, the HE machines are categorized into two different groups, which are series HE machines and parallel HE machines. For series HE machines, the flux created by the field coils would go through the PMs. Thus PM and field winding have the same flux path and on the other hand in case of parallel, HE machines the fluxes of both excitations travel in separate paths hence there are fewer chances of demagnetizations and cancellation.[18].

Therefore, a new structure of 12S-8P HE-FSM with segmental rotor has been proposed and investigated in this paper shown in Figure. 1. In which PM and FE are used as primary and secondary excitation sources and generating the parallel hybrid excitation to avoid cancellations and demagnetization effects in between FE and PM fluxes. Besides, coil test analysis has been performed to validate the working principle of the motor and the performance analysis with no-load and load conditions are also examined on the basis 2-D FEA

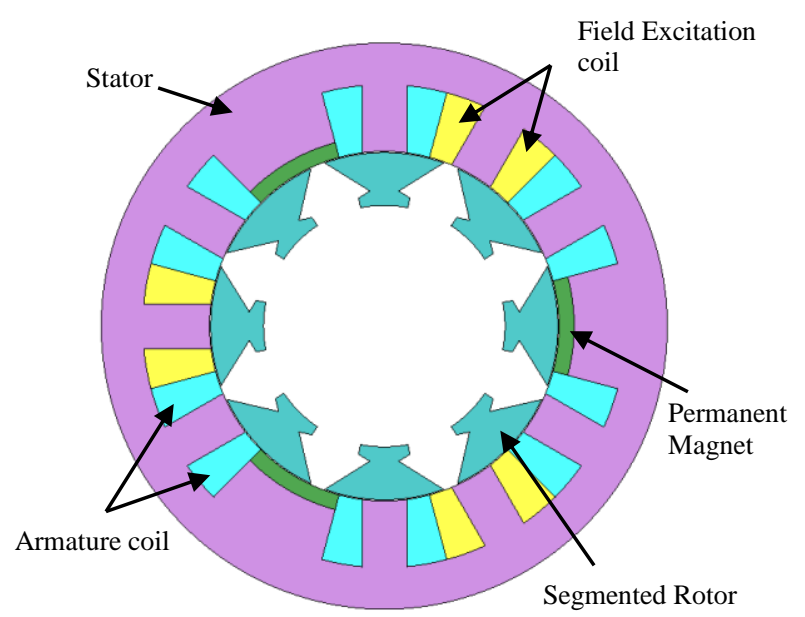

Fig. 1: 12S-8P HE-FSM segmental rotor

\section{Design Parameters, Specification and Methodology}

The design parameters and design specifications of a new structure of HE-FSM with segmented rotor designed using JMAG designer, are illustrated in Table 1 . This study is divided into two sections, which are geometry editor and JMAG Designer. Each part of the motor is designed separately using geometry editor, such as the stator, rotor, armature coil, PM, and FEC. While the materials settings, conditions settings, circuit design, mesh, and simulation setting are developed by using the JMAG Designer. In the proposed segmented HE-FSM, the probable number of rotor pole and stator slot is defined by Equation (1), which has an intense role in designing the optimize structure of the machines.

$$
N_{r}=N_{s}\left(1 \pm \frac{k}{2 q}\right)
$$

Where $\mathrm{Nr}$ represented a rotor pole number, $\mathrm{Ns}$ is the number of stator slot, $\mathrm{k}$ is the integer from 1 to 5 and $\mathrm{q}$ is the number of phases.

By using the same parameter restriction and specification of 12 slot- 8 pole HE-FSM, the performances are analysed based on 2D-FEA for open circuit conditions which mean no current is supplied to armature coil and also in load condition with maximum armature coil and DC-FEC current densities of $30 \mathrm{Arms} / \mathrm{mm}^{2}$ and $30 \mathrm{~A} / \mathrm{mm}^{2}$, respectively.

\section{Performance analysis}

In this section, performance of new structure of HE-FEM has been carried out at no load as well as at full load conditions by using JMAG designer on the basis of 2D-FEA. Firstly, at no load coil test analysis, cogging torque and back emf have been investigated to verify the basic working principle of machine. Secondly, at load conditions, performance has been investigated in terms of torque and power.

\subsection{Coil test analysis}

Coil arrangement test analysis is examined in each armature coil separately in order to validate the operating principle of the $12 \mathrm{~S}$ 8P segmented HE-FSM, and to endorse the position of each armature coils phase. Thus, the DC current of 51.27A is applied to the FE coil plus inward direction PMs employed to produce the flux linkage in the armature coil. The flux linkage is observed in each armature coil at different rotor positions from 0 to $360^{\circ}$ as illustrated in Figure. 2. From figure, it is shown that all armature coils 
are organized in the alternate directions. Consequently, the three phase flux linkage defined as $\mathrm{U}, \mathrm{V}$, and $\mathrm{W}$ with maximum flux linkage of $0.056 \mathrm{~Wb}$ has accomplished as revealed. With this arrangement, it can be seen that proposed machine has achieved sinusoidal waveform which confirms the operating principle of the machine as shown in Figure. 3.

Table 1: Design Parameters and Specifications of HE-FSM

\begin{tabular}{lc}
\hline \multicolumn{1}{c}{ Items } & (HE-FSM) with \\
& Segmental rotor \\
\hline Number of stator slots & 12 \\
Number of segments of rotor & 8 \\
Outer radius of stator (mm & 75 \\
Back inner width of stator (mm) & 11 \\
Tooth width of stator (mm) & 12.5 \\
Slot area armature coil (mm ${ }^{2}$ ) & 250 \\
Slot area of FEC (mm $\left.{ }^{2}\right)$ & 250 \\
Outer radius of rotor (mm) & 45 \\
Inner radius of rotor (mm) & 30 \\
Length of air gap (mm) & 0.3 \\
Segment span & $40^{\circ}$ \\
Number of turns per slot of FEC & 44 \\
Number of turns per armature coil (AC) slot & 44 \\
\hline
\end{tabular}

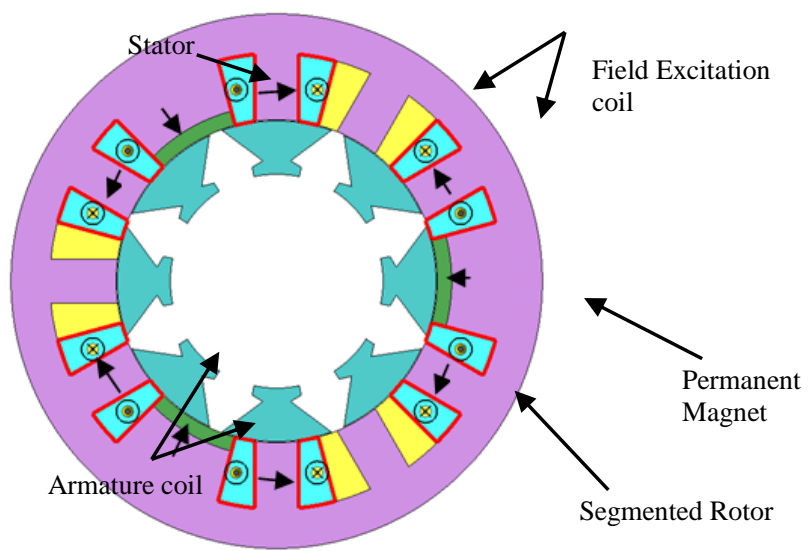

Fig.2: Arrangement of Armature coil for HE-FSM with segmental rotor

\subsection{Cogging torque at zero load condition}

The cogging torque or unwanted torque, it is also called as detent or no-current torque, which is the key source to create noise and vibrations in machine operations specifically in PM machines. Therefore, it the foremost objective of the researcher to reduce cogging torque until safe values. Additionally, in the proposed HE-FSM design, the weight and number of PM is reduced to limit the cogging torque.

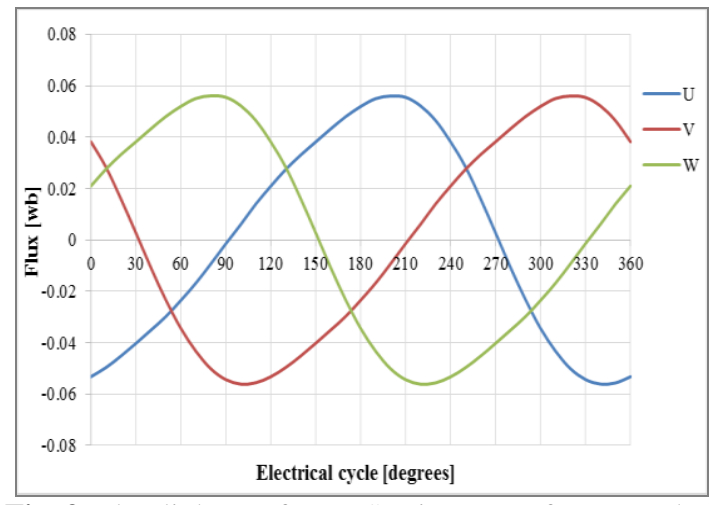

Fig. 3: Flux linkage of HE-FSM in terms of U, V, and W

Figure. 4. Illustrates the no load torque of three phase HE-FSM with segmental rotor. From the figure, it is noticeable that HEFSM with segmental rotor has less peak to peak value of cogging torque about $4.021 \mathrm{Nm}$. From the analysis, it is observed that HEFSM with segmental rotor has achieved the healthier result of less cogging torque due to the simple structure as there are only three FECs and three PM used leaving much free space in the stator. Less cogging torque results that motor will work at high speed without vibrations, and avoid acoustic noises. Which is appropriate for aircraft applications where the motor with less weight and fewer vibrations is obligatory.

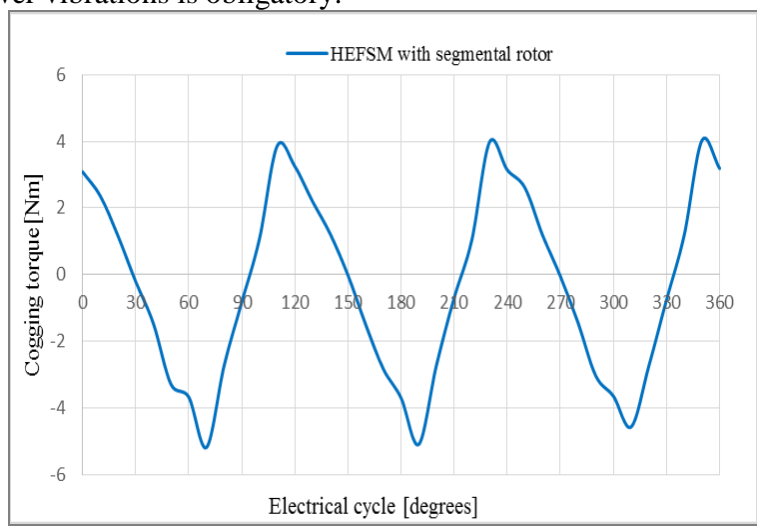

Fig. 4: Cogging torque of HE-FSM with segmental rotor

\subsection{Magnetic flux strengthening}

Magnetic flux strengthening of a new structure of HE-FSM with a segmental rotor has been carried out on the basis 2-FEA at zero armature current density ( $\mathrm{Ja}$ ) to examine the behaviour of both excitation sources. Figure 5 shows the flux strengthening of proposed structure in which maximum strengthening is achieved approximately $0.065 \mathrm{~Wb}$ at the maximum field current density (Je) of $10 \mathrm{~A} / \mathrm{mm}^{2}$, where the fluxes of both sources (FE \& PMs) are combined properly. Furthermore, magnetic flux strengthening is slightly decreases as the current density is increased due to the reason of flux saturation in the stator. There is some flux saturation at the tips of stator poles due to the maximum amount of flux is produced, However, by further optimization and design refinement, fluxes from both sources FE coil and PMs will be combined easily at maximum current densities. Besides, flux will be transferred from stator to rotor segments smoothly and able to generate more magnetic flux strengthening.

\subsection{Magnetic flux distribution and flux lines}

Analysis of magnetic flux distribution and magnetic flux lines have been conducted at no load conditions. Where armature current density has been applied is zero, field current density applied is $30 \mathrm{~A} / \mathrm{mm}^{2}$, whereas $P M$ is producing constant flux linkages. Moreover, Magnetic flux distribution and magnetic flux lines of new segmented HE-FSM are illustrated in Figure. 6 and Figure. 7 respectively. It is obvious from the figure 6 , that flux is distributed smoothly and properly over the stator and the rotor segments due to enough space between stator slots. On the other hand segmented rotor confirms the shortest flux path between one stator slot to another slot as shown in Figure. 6.

Furthermore, Fig. 7 illustrates the magnetic flux lines, which are properly and continuously flowing through stator to the rotor segments without cancellation and making proper and short completed cycles. By using only three PMs and three FECs, stator iron core gets much free space which gives the advantage of full utilization of flux linkages over the stator and reducing the saturation and flux cancellation between fluxes from both sources. 


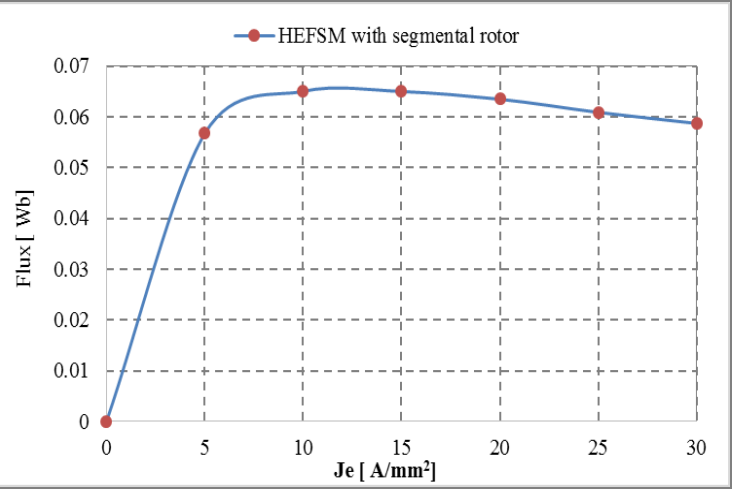

Fig. 5: Magnetic flux strengthening of HE-FSM with segmental rotor

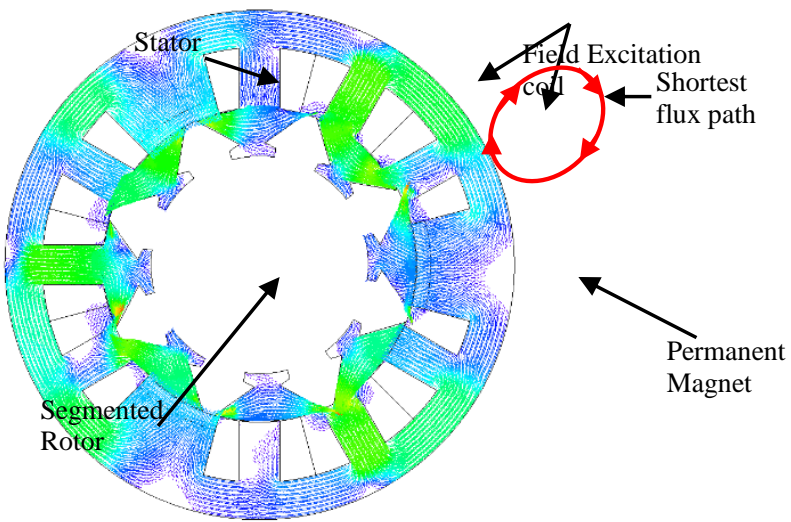

Fig. 6: Magnetic Flux distribution of segmented HE-FSM

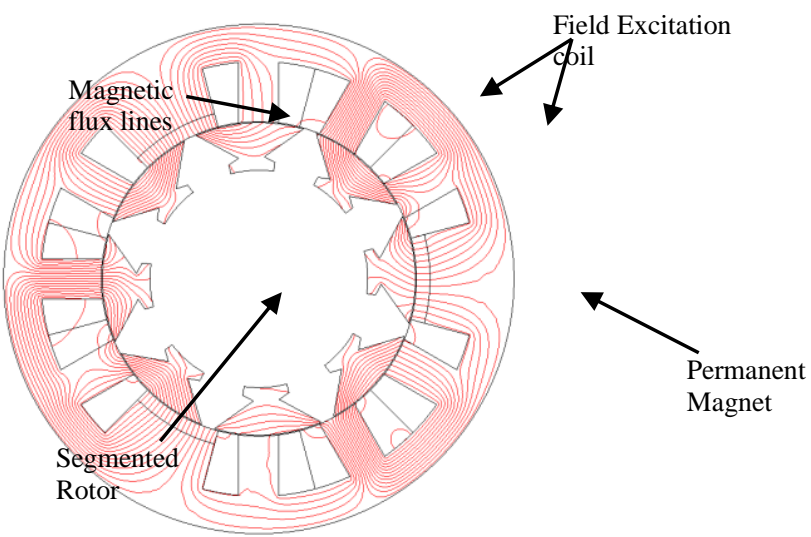

Fig. 7: Magnetic Flux lines of segmented HE-FSM

\section{Torque Vs Je, at different armature current densities of Ja}

Torque vs. field excitation current densities Je, at various armature current densities Ja, of HE-FSM with the segmental rotor is shown in Figure. 8. From the figure, it is observable that the torque value is increasing directly with the increase of field current density (Je), and reaches the maximum value of approximately $36.68 \mathrm{Nm}$ when the maximum armature current density of $30 \mathrm{Arms} / \mathrm{mm}^{2}$ has been applied. It is clear from the figure that the new structure of HEFSM with segmental rotor has achieved the maximum value of torque than the previous designs of HE-FSM and confirms that the fluxes of FECs and PMs are combined properly due the enough space in the stator with smooth distribution and better arrangement of both excitation sources. From the figure, it is also noticeable that with further increase of current densities the torque is increased directly, it confirms that there is no any magnetic saturation or flux cancellation is produced. Due to the high torque, simple structure and light weight the proposed structure is reliable for aircraft applications such as actuation, engine fuel pump operation and for the propeller. The torque of new design further can be increased up to the optimum value by design refinement and optimization.

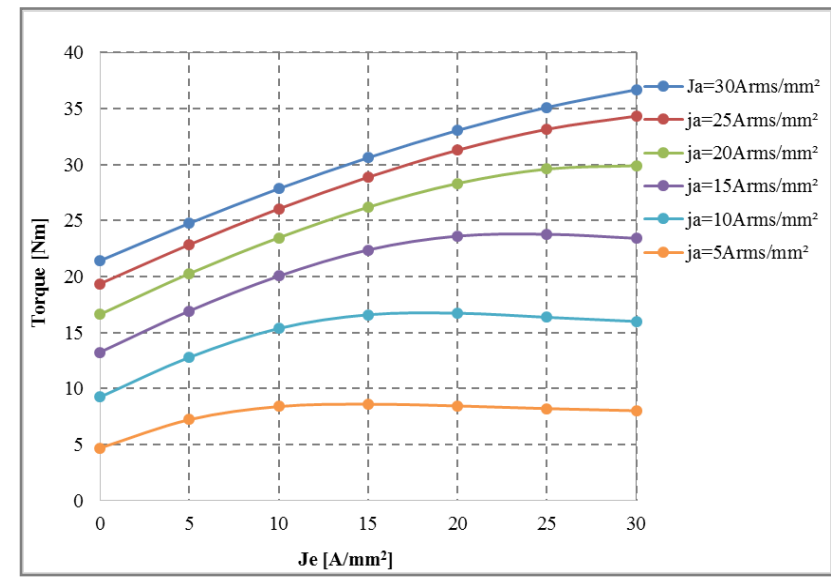

Fig. 8: Torque vs Je of HE-FSM with segmental rotor

\section{Power vs armature current densities Ja}

Power vs armature current densities Ja at maximum current density Je of $30 \mathrm{~A} / \mathrm{mm}^{2}$ for HE-FSM with the segmental rotor is illustrated in Figure. 9. From the figure, it is obvious that the power of the new structure of the HE-FSM structure increases linearly as the armature current density is increased which shows that there is negligible cancellation of fluxes between both excitation sources. Furthermore, at speed of $500 \mathrm{rpm}$, the HE-FSM has achieved the maximum value of power approximately $15.4 \mathrm{~kW}$ at maximum armature density of $30 \mathrm{Arms} / \mathrm{mm}^{2}$. As there are only three FE coils are used, therefore, it causes the reduction of copper cost as well as copper losses. Hence, results maximum power has been achieved which is beneficial for aircraft applications especially for the aircrafts at lower altitudes. The aircrafts flying at lower altitudes for specific applications require more torque and power to carry the load. For lower altitude aircrafts, the proposed HE-FSM fulfills the requirements to be the best candidate, which not only has gained high performances but can be work in fault conditions as there are two separate excitations are used. Furthermore, the proposed HE-FSM with segmental rotor is able to attain more torque until optimum values by design refinement and optimization techniques.

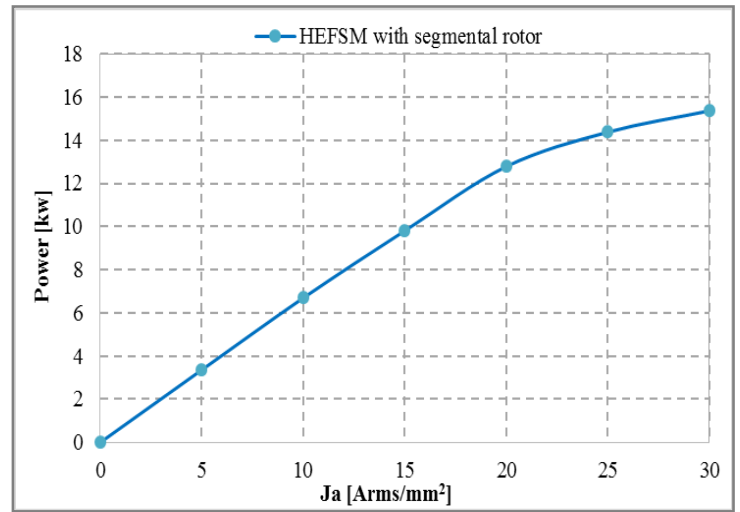

Fig. 9: Power vs Ja of HE-FSM with segmental Rotor at maximum Je

\section{Conclusion}

In this paper, the design and performance analysis of the new structure of HE-FSM using segmental rotor has been introduced. The proposed design contains only three number of FECs and three PMs which causes the less weight and less cost of the motor. Initially, the coil test analysis of new structure has been investigated to confirm the working principle with segmental rotor based on 2-D FEA. As the new structure uses only three FECs and three 
PMs to confirm the simple structure and smooth flow of magnetic fluxes along the stator to rotor segments. Besides this, three FECs con-firms the lesser copper losses as compared to the existing designs. Furthermore, cogging torque, flux strengthening have been analysed and validated that the new structure will work in the safe regions with adequate results. From the analysis of new structure, it is obvious that the proposed design of HE-FSM with the segmental rotor is the best candidate for the low altitude aircraft applications as the motor draws high torque and high power at maximum speeds ranges. Furthermore, the new structure is able to attain much more torque and power performances by design refinement and optimization techniques.

\section{Acknowledgement}

This research work was promoted by Research, Innovation, Commercialization and Consultancy (ORICC) UTHM, Batu Pahat under Vot number (FRGS) 1548 and Ministry of Higher Education Malaysia (MOHE).

\section{References}

11] Wenping. C, Mecrow B. C, Glynn J., John W. B, David J. A "Overview of Electric Motor Technologies Used for More Electric Aircraft (MEA)", IEEE Transactions on Industrial Electronics, Vol. 59, No.9, (2012), pp. 3523-3531.

[2] A. Boglietti, A. Cavagnino, A. Tenconi, and S. Vaschetto, "The safety critical electric machines and drives in the more electric aircraft: A survey", IEEE Conference of Industrial Electronics, (2009), pp. 2587-2594.

[3] B. K. Bose, "Power electronics and motor drives-Recent progress and perspective", IEEE Trans. Ind. Electron., Vol. 56, No. 2 , (2009), pp. 581-588.

[4] A. C. Hoffman, I. G. Hansen, R. F. Beach, R. M. Plencher, R. P Dengler, K. S. Jefferies, and R. J. Frye, "Advanced Secondary Power System for Transport Aircraft", Washington, DC: NASA, ser. NASA Technical Paper 2463. (1985).

[5] G. J. Atkinson, B. C. Mecrow, A. G. Jack, D. J. Atkinson, P. Sangha, and M. Benarous, "The analysis of losses in high-power faulttolerant machines for aerospace applications", IEEE Trans. Ind. Appl., Vol. 42, No. 5, (2006), pp. 1162-1170.

[6] B. K. Bose, "Power electronics and motor drives recent progress and perspective", IEEE Trans. Ind. Electron., Vol. 56, No. 2, (2009). pp. 581-588.

[7] S. Dwari and L. Parsa, "Fault-tolerant control of five-phase permanent magnet motors with trapezoidal back EMF", IEEE Trans. Ind. Electron., Vol. 58, No. 2, (2011), pp. 476-485.

[8] E. Levi, "Multiphase electric machines for variable-speed applications", IEEE Trans. Ind. Electron., Vol. 55, No. 5, (2008), pp. 1893-1909.

[9] N. Bianchi and S. Bolognani, "Fault-tolerant PM motors in automotive applications", in Proc. IEEE Conf. Vehicle Power Propulsion, (2005), pp. 747-755

[10] R. Krishnan and A. S. Bharadwaj, "A comparative study of various motor drive systems for aircraft applications", in Conf. Rec. IEEE IAS Annul. Meeting, 1991, Vol. 1, pp. 252-258, (1989).

[11] Sulaiman, E. Kosaka, T. and Matsui, N. "A New Structure of 12Slot-10Pole Field-Excitation Flux Switching Synchronous Machine for Hybrid Electric Vehicles", Proceedings of 14th European Conferences on Power Electronics and Applied. (EPE), UK, Paper No.362, (2011)

[12] Soomro, H.A. Sulaiman, E. Omar, M.F, "Performance Comparison and analysis of (HE-FSM) and (FEFSM) using Segmental rotor Structure', Applied Mechanics and Materials, Vol.695, (2015), pp. 778-782.

[13] C. Sanabria-Walter, H. Polinder, and J. A. Ferreira, "HighTorque Density High-Efficiency Flux-Switching PM Machine for Aerospace Applications", IEEE Trans. Emerg. Sel. Topics Power Electron, Vol. 1, No. 4, (2013), pp. 327-336.

[14] I. Boldea, L. N. Tutelea, L. Parsa, and D. Dorrell, "Automotive electric propulsion systems with reduced or no permanent magnets: An overview", IEEE Trans. Ind. Electron., Vol. 61, No. 10, (2014) pp. 5696-5711.

[15] Z. Q. Zhu, Z. Wu, D. J. Evans, W. Q. Chu, "A Wound Field Switched Flux Machine With Field and Armature Windings Sepa- rately Wound in Double Stators," IEEE Transactions on Energy Conversion, Vol. 30, No. 2, (2015), pp. 772-783.

[16] F. Capponi, G. Borocci, G. Donato, and F. Caricchi, "Flux regulation strategies for hybrid excitation synchronous machines", IEEE Trans. Ind. Appl., Vol. 51, No. 5, (2015), pp. 3838-3847.

[17] Y. Amara, L. Vido, M. Gabsi, E. Hoang, A. Ahmed, and M. Lecrivain, "Hybrid excitation synchronous machines: energyefficient solution for vehicles propulsion", IEEE Trans. Veh. Technol., Vol. 58, No. 5, (2009), pp. 2137-2149.

[18] H. Lin, X. Liu, Z. Q. Zhu, and S. Fang, "Analysis and control of a dual stator hybrid excitation synchronous wind generator", IET Electr. Power Appl., Vol. 5, No. 8, (2011), pp. 628-635. 